\title{
Principles of calibrating the dual-recycled GEO 600
}

\author{
M. Hewitson, ${ }^{\text {a) }}$ G. Heinzel, and J. R. Smith \\ Max-Planck-Institut für Gravitationsphysik (Albert-Einstein-Institut) und Universität Hannover, \\ Außenstelle Hannover, Callinstr. 38, 30167 Hannover, Germany
}

K. A. Strain and H. Ward

Physics and Astronomy, University of Glasgow, Glasgow, G12 8QQ Great Britain

(Received 26 April 2004; accepted 7 July 2004; published 1 November 2004)

\begin{abstract}
GEO 600 is a dual-recycled interferometric gravitational wave detector which uses advanced optical techniques to achieve its design sensitivity. The peak response of GEO is tunable over a range of frequencies within the detection band of the instrument. The accurate calibration of the detector is an essential step in preparing the data for distribution to the various analysis groups in the community as well as allowing for the extraction of science results. Calibrating the dual-recycled interferometer requires the optical response of the instrument to be determined and corrected for on time scales associated with any fluctuations in the response. Due to the optical readout scheme used in GEO 600, any gravitational wave signals seen by the detector are split between two output signals. This means that, in order to recover a calibrated strain signal that has an optimal signal-to-noise ratio, the two outputs need to be combined pre- or postcalibration. This article presents a detailed method for calibrating the dual-recycled GEO configuration. The method builds upon the calibration scheme developed to calibrate the power-recycled GEO configuration. The main focus of this article is on the new aspects of the calibration process: on-line measurement, parameterization, and correction of the optical response of the instrument in an on-line, time-domain calibration pipeline. Some discussion is also given regarding the combination of the two main detector output signals in producing an optimal calibrated strain signal. (C) 2004 American Institute of Physics. [DOI: 10.1063/1.1804831]
\end{abstract}

\section{INTRODUCTION}

The GEO 600 interferometric gravitational wave detector is a German-British detector located outside Hannover, Germany. By using a combination of signal and power recycling, GEO 600 will, when fully commissioned, be one of the most sensitive displacement measuring instruments in the world, having the ability to measure differential arm-length changes between the two $1200 \mathrm{~m}$ long arms of the order $10^{-19} \mathrm{~m} / \sqrt{\mathrm{Hz}}$ in the frequency range from 50 to $6000 \mathrm{~Hz}$.

Commissioning of GEO 600 has been done on two main detector configurations: a power-recycled only configuration, and the dual-recycled configuration. The power-recycled Michelson configuration was commissioned prior to installation of the signal-recycling mirror to allow for the many subsystems in GEO to be debugged with a simpler optical configuration. After a long period of working on the powerrecycled configuration, the signal-recycling mirror was installed and a further extended period of debugging and commissioning followed. During this time, a new locking scheme for the dual-recycled (DR) interferometer was developed and tested. Details of the commissioning of GEO during this time are given in Ref. 1.

The optical layout of the dual-recycled interferometer is based on the Michelson interferometer with the addition of a

\footnotetext{
${ }^{\text {a) }}$ Author to whom correspondence should be addressed; electronic mail: martin.hewitson@aei.mpg.de
}

power-recycling (PR) mirror placed at the input port, and a signal-recycling (SR) mirror placed at the output port of the detector. The addition of the power-recycling mirror forms a cavity that increases the power circulating in the interferometer, leading to improved shot-noise performance of the detector, while the signal-recycling mirror forms an optical cavity with the Michelson interferometer which resonantly enhances the signal sidebands, and hence, any gravitational wave signals that are present. The principles of signalrecycling are covered in detail in Refs. 2-5.

The presence of the signal-recycling cavity means that the signal transfer function from differential mirror displacement to detector output signal is no longer flat in the frequency domain (as in the PR configuration). This means that, in order to recover an optimal calibrated strain signal from the raw detector outputs, we need to determine many more parameters that describe the optical response. The response of the cavity (and thus, the parameters) depends on the microscopic position, or tuning, of the SR mirror and on the mirror reflectivity. Fluctuations in the optical response can arise, for example, from alignment drifts due to tidal, seismic, and thermal effects, as well as from fluctuations in the input laser power. In the power-recycling configuration of GEO 600, fluctuations in the optical power of the order of $20 \%$ were observed on daily and faster time scales; such fluctuations can also be expected for the dual-recycled configuration of GEO.

If such fluctuations are not accounted for, they translate 
directly into an error in the calibration. In other words, if the overall optical gain of the detector were to vary $20 \%$ on daily time scales, due to, for example, temperature, and the calibration used a constant value for the overall optical gain, then we would have an error in the calibration of up to $20 \%$. In order to account for such drifts in the calibration process, the various parameters that describe the detector response need to be continually estimated. The calibration of the dualrecycled GEO is, therefore, significantly more complicated than that of the power-recycled GEO (where the calibration is given basically by one number).

As in the case of calibrating the power-recycled GEO, the calibration of the dual-recycled GEO is best approached in the time domain so that these fluctuations in the optical response of the detector can be smoothly corrected for. The calibration scheme used for the power-recycled configuration of GEO is detailed in Ref. 6 and shown applied to an extended science run in Ref. 7. This article presents a timedomain calibration scheme for recovering the strain signal, $h(t)$, from the output signal(s) of the dual-recycled GEO interferometer. This calibration scheme builds upon the methods used for calibration the power-recycled GEO.

\section{EXTENDING THE CALIBRATION OF THE POWER-RECYCLED GEO 600}

Work following the development of the power-recycled GEO calibration scheme identified a few areas of possible improvement. As well as the additional methods required to calibrate the dual-recycled GEO 600, two of these possible improvements are discussed later after a short review of the power-recycled GEO calibration scheme.

\section{A. Review of the power-recycled GEO 600 calibration scheme}

Calibration of the power-recycled GEO 600 configuration is done by calibrating out the two main transfer functions that affect the signal transfer from differential armlength changes to detector output: one of these is the optical response of the instrument; the other concerns the control scheme used to keep the detector at its correct operating point.

\section{The Michelson locking scheme}

The design of GEO is such that it is most sensitive when it is held at on a dark fringe at the detector output port. However, any external disturbances from, for example, seismic noise will move the detector away from this optimal operating point. For the purpose of signal readout and control modulation, sidebands are imposed on the carrier light injected into the interferometer. The light detected at the output port can be appropriately demodulated to give a control signal that reflects any deviations from the dark fringe. This signal is used to feed back through appropriate electronic filters to length-control actuators on the two arms of the detector. Thus, the detector is held very close to the dark fringe operating point. The error signal of the Michelson lengthcontrol servo loop forms the main output of the detector and contains, at most frequencies in the detection band, any po- tential gravitational wave signals. Since this control system aims to suppress any deviations from the dark fringe that occur at frequencies within the bandwidth of the servo system, any gravitational wave signals that are present at those frequencies will also be suppressed. The feedback control system is discussed in more detail in the context of calibrating the power-recycled GEO configuration in Ref. 6.

\section{Calibration principles}

In order to correct for the optical response of the instrument and for the effect of the Michelson length-control servo, we need to be able to estimate the transfer function of each of them. By injecting calibration signals into the lengthcontrol actuator, the effect of both of these transfer functions can be estimated. For the case of calibrating the powerrecycled interferometer, the calibration lines were injected at (high) frequencies where the effect of the Michelson servo was negligible. This allowed the (flat) optical gain of the instrument to be estimated periodically. Having estimated the optical gain, it can be inverted and used to correct the recorded error-point signal. Having done this we have a signal, $d x_{\text {opt }}$, that, at least at frequencies well above the unity gain point of the Michelson servo, represents the differential armlength changes of the detector. At lower frequencies where the Michelson servo has non-negligible loop gain, we must generate a representation of the feedback signal(s), $d x_{\mathrm{fb}}$, and add it to $d x_{\text {opt }}$. Figure 1 shows a schematic of the Michelson length-control servo and how it can be used to calibrate the detector output signal by inverting the loop transfer function in software.

The recovered differential displacement signal can then be converted to a strain according to the following equation:

$$
h(t)=2 \frac{\Delta L(t)}{L},
$$

where $\Delta L$ is the length change of one arm of the interferometer, and $L$ is $1200 \mathrm{~m}$ for GEO. In summary, the calibration of the power-recycled GEO can be viewed as the determination of $\Delta L$ from the detector output, i.e., the determination of the detector gain, as a function of frequency, in volts per meter.

\section{B. Improvements carried forward}

One of the possible improvements of the time-domain calibration scheme for the power-recycled GEO is in the method used to up-sample the overall optical gain estimates. Because the overall optical gain is estimated at only $1 \mathrm{~Hz}$ (due to the limited signal-to-noise ratio of the calibration lines in the detector error-point signal), we need to interpolate the estimates to the sample rate of the recorded errorpoint signal $(16384 \mathrm{~Hz}$ ). Previously, a spline interpolation method was used to smoothly correct the detector output for changes in the overall optical gain of the instrument by allowing the optical gain estimates to be calculated at each sample instant of the detector output signal. This technique was a quick solution that was implemented in order to conform to the time constraints of the experiment. The process of up-sampling the $1 \mathrm{~Hz}$ optical gain estimates to $16384 \mathrm{~Hz}$ can be done in another way: using a band-limited interpola- 


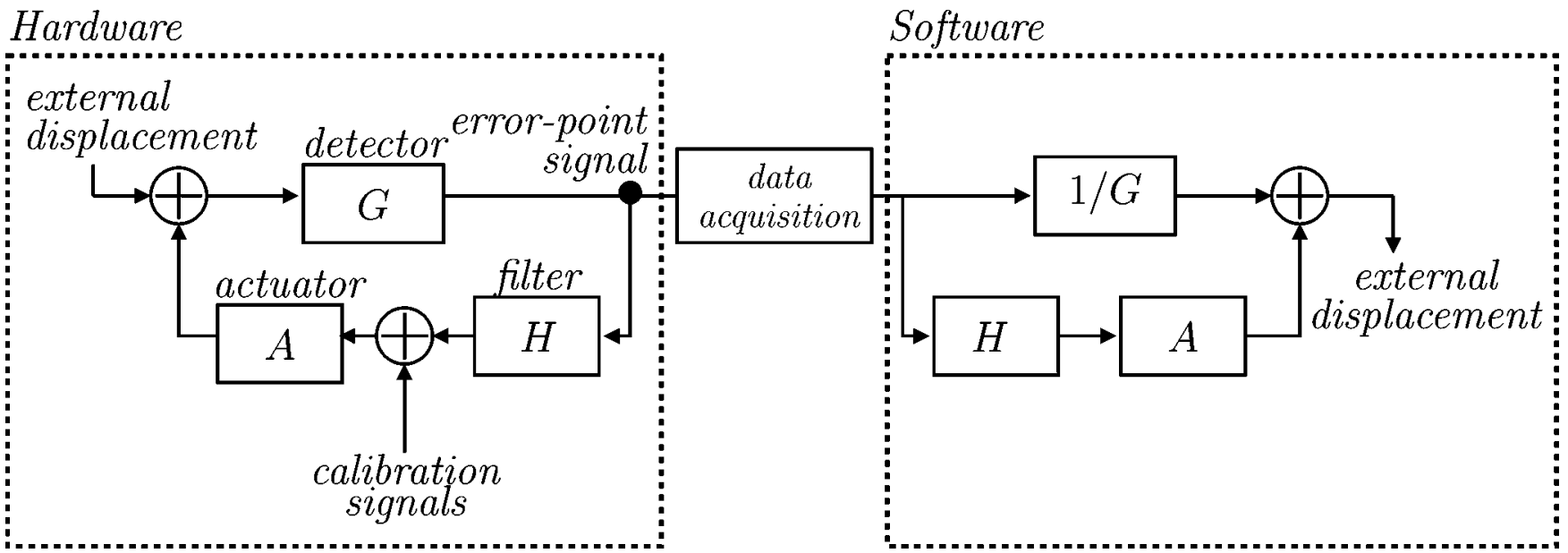

FIG. 1. A schematic overview of the underlying calibration principle used for calibrating the power-recycled GEO 600. The feedback system in GEO (shown here as one path for simplicity) is actually split between a low-frequency actuator and a high-frequency actuator.

tion method. This method takes into account the implicit assumption that the optical gain does not vary on time scales shorter than two seconds by applying a suitable low-pass filter. (This assumption arises because we sample the optical gain at only $1 \mathrm{~Hz}$.) This method can also be used for upsampling the detector output signal prior to some parts of the signal processing pipeline (see Sec. III B).

In the power-recycled calibration scheme, the optical gain was estimated once per second and the subsequent samples were smoothed using a Hanning window of length $60 \mathrm{~s}$. This was done to reduce effect of any transients in the estimated optical gain samples as it was felt that faster fluctuations could not be measured well enough to be adequately corrected. The Hanning window smoothing technique is replaced in the DR calibration scheme with a two pole IIR low-pass filter with corner frequency at $0.1 \mathrm{~Hz}$.

\section{Modeling the optical response of the dual-recycled GEO 600}

In the power-recycled setup of GEO, the optical gain was assumed to be flat across the detection band of the instrument (see Ref. 6). The introduction of a signal-recycling mirror means that this is no longer the case and so we need to determine, and correct for, the now nonflat (resonant) response of the detector to differential arm-length changes. An additional complication arises because the optimal demodulation phase for the output photodiode is no longer independent of frequency as it was for the power-recycled optical configuration. This means that signals are spread between the two quadratures of the demodulated output photodiode. By selecting a particular demodulation phase, we maximize signals of a particular frequency in one quadrature and minimize them in the other; signals at other frequencies appear with different ratios in the two quadratures. In order to use the output photodiode as the loop sensor for the Michelson length-control servo, we would typically choose a demodulation phase that maximises one quadrature at low frequencies to give large signals at the frequencies where the feedback is most dominant. This "maximized" quadrature will be referred to here as the in-phase or $\mathbf{P}$ quadrature; the other quadrature is termed out-of-phase or $\mathbf{Q}$. More generally, these terms are used for all demodulation phases with the general definition that the signal content in $\mathbf{P}$ is maximum (and minimum in $\mathbf{Q}$ ) at a chosen frequency.

Using the interferometer simulation software, FINESSE, ${ }^{8}$ the response of the signal-recycling cavity to differential arm-length changes can be modeled. Figure 2 shows the response of the signal-recycling cavity with a mirror reflectivity of $99 \%$ and microscopic tuning of around $1200 \mathrm{~Hz}$. Both demodulation quadratures are shown. The demodulation phase is chosen in this simulation to give a minimum response of the $\mathbf{Q}$ quadrature at direct current (dc). As we can see, setting the phase like this means that the demodulation at all other frequencies is nonoptimal and signal power is spread between the two demodulation quadratures. To recover the full signal with optimal signal-to-noise ratio, we need to consider both of the demodulation quadratures. We can also see that the optical gain parameter, $H_{1}$, introduced in Ref. 6, is no longer independent of frequency in the detection band. So we introduce two new parameters, $H_{\mathbf{P}}$ and $H_{\mathbf{Q}}$, which represent the transfer functions from differential mirror motion to the two demodulated output signals, $\mathbf{P}$ and $\mathbf{Q}$; both of these need to be determined to allow for an optimal reconstruction of the calibrated strain signal.

\section{Parameterizing the optical response}

If we are to correct for the optical response of the signalrecycling cavity as measured in both quadratures, then we need to be able to model the two responses. For the purpose of modeling the system, it is convenient to consider the two demodulation quadratures as separate transfer functions from differential mirror displacement to the detector output signals.

Simulations of the response of the dual-recycled interferometer to differential arm-length changes were generated using a FINESSE model of the detector. The response of the two quadratures can be modeled with one complex pole and one real zero together with a suitable gain factor. The frequency of the pole depends on the microscopic detuning of the SR mirror, and its quality factor, $Q$, is related to the 

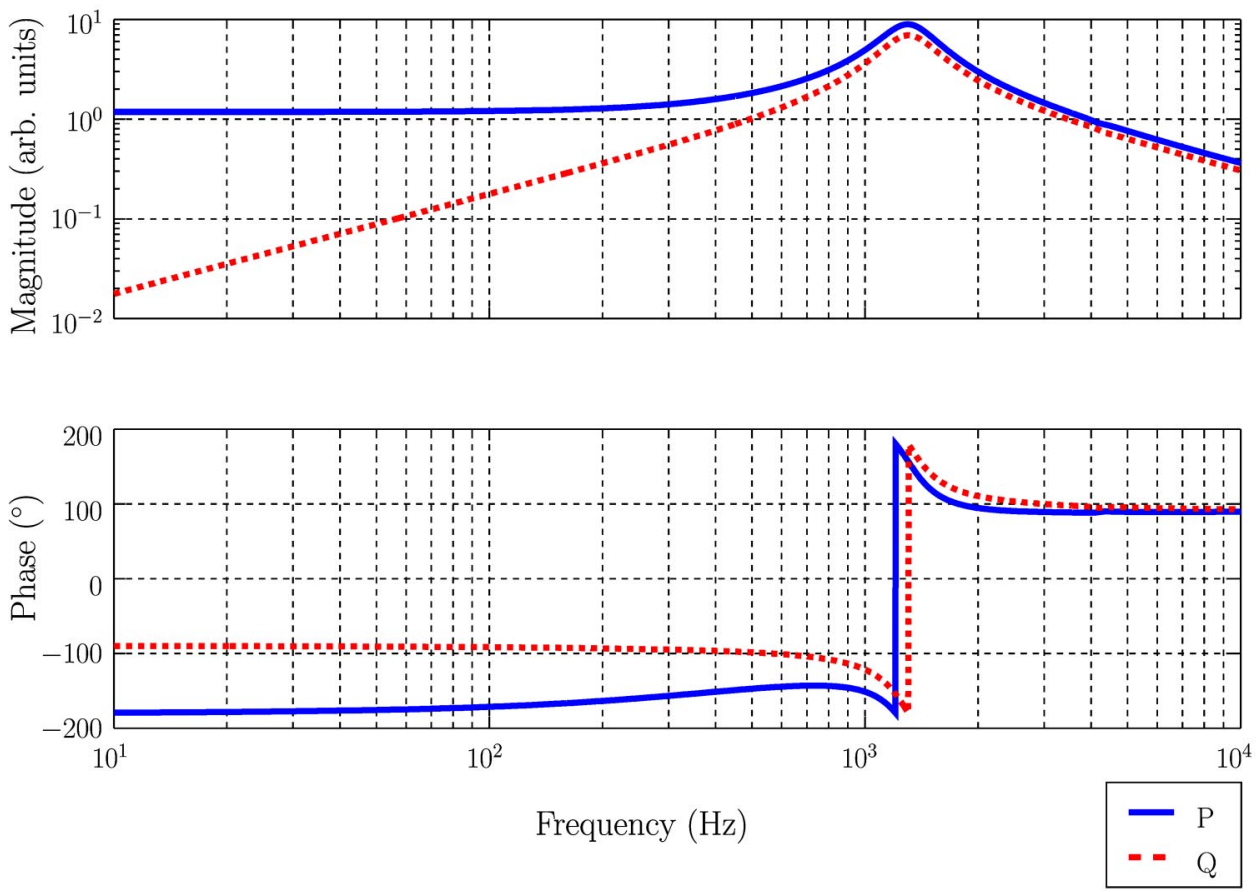

FIG. 2. (Color online) The in-phase and out-of-phase responses of the signal-recycling cavity to differential arm-length changes. The response is shown for a detuning of around $1200 \mathrm{~Hz}$ with a SR mirror reflectivity of $99 \%$. The demodulation phase is set to give optimal signal size in $\mathbf{P}$ at $0 \mathrm{~Hz}$.

bandwidth of the cavity (which is determined by the reflectivity of the signal-recycling mirror). The pole $Q$ is also weakly related to the microscopic tuning of the signalrecycling mirror. Suitable models of the two optical responses are

$$
\begin{aligned}
& H_{\mathbf{P}}(s)=G_{\mathbf{P}} \frac{\left(s-z_{\mathbf{P}}\right)}{\left(s-p_{\mathbf{P}}\right)\left(s-p_{\mathbf{P}}^{*}\right)}, \\
& H_{\mathbf{Q}}(s)=G_{\mathbf{Q}} \frac{\left(s-z_{\mathbf{Q}}\right)}{\left(s-p_{\mathbf{Q}}\right)\left(s-p_{\mathbf{Q}}^{*}\right)},
\end{aligned}
$$

where "**" denotes complex conjugation, $z_{\mathbf{P}}$ is the zero in the $\mathbf{P}$ response, $z_{\mathbf{Q}}$ is the zero in the $\mathbf{Q}$ response, and so on. Looking again at Fig. 2, we see that the $\mathbf{P}$ transfer function has a pole at around $1200 \mathrm{~Hz}$ and a zero at higher frequency, whereas the $\mathbf{Q}$ transfer function has a pole at approximately the same frequency but with a zero at dc.

These equations can be parameterized in a more convenient way using a frequency/ $Q$ description of the poles and zeros. In doing this, we have eight parameters to determine in order to characterize the response of the two demodulated optical signals to differential mirror displacement; these are, for each of $\mathbf{P}$ and $\mathbf{Q}$, an overall gain factor, a pole frequency, a pole quality factor, and a zero frequency.

\section{Obtaining the optimal signal-to-noise ratio strain signal}

There are two main issues regarding the recovery of the strain signal with optimal signal-to-noise ratio. One of the points is that, for a low-noise detector, it may be necessary to recover the gravitational wave signal from both the feedback and the error-point signals of the Michelson control servo in order to maintain an optimal signal-to-noise ratio (SNR) dur- ing the data acquisition process. For the dual-recycled GEO, this may well be the case, especially as the detector comes closer to its design sensitivity.

An additional consideration arises because of the frequency dependent optimal demodulation phase (see Fig. 3). At a particular frequency, a gravitational wave signal will appear in both quadratures. In the absence of noise, the full underlying gravitational wave signal can be recovered by calibrating either of these quadrature signals back to a displacement signal. However, in the presence of noise, the situation is not as clear; we can still calibrate both demodulated output signals back to displacement but, depending on the frequency of interest, the optimal signal-to-noise ratio may only be gained by a suitable combination of the two recovered displacement signals. Depending on where noise is
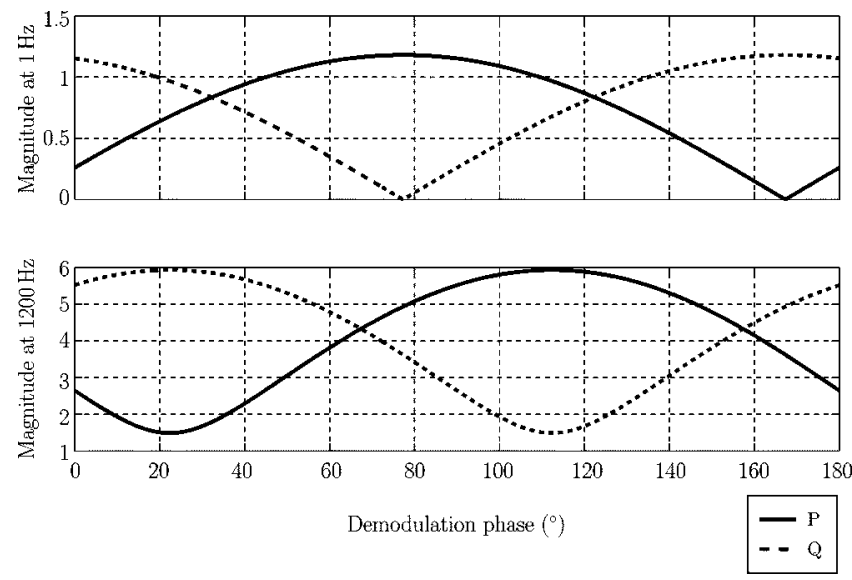

FIG. 3. Michelson output photodiode demodulation phase curves for two signal frequencies. Using such curves, the demodulation phase can be chosen such that $\mathbf{P}$ is maximum at the chosen signal frequency. 


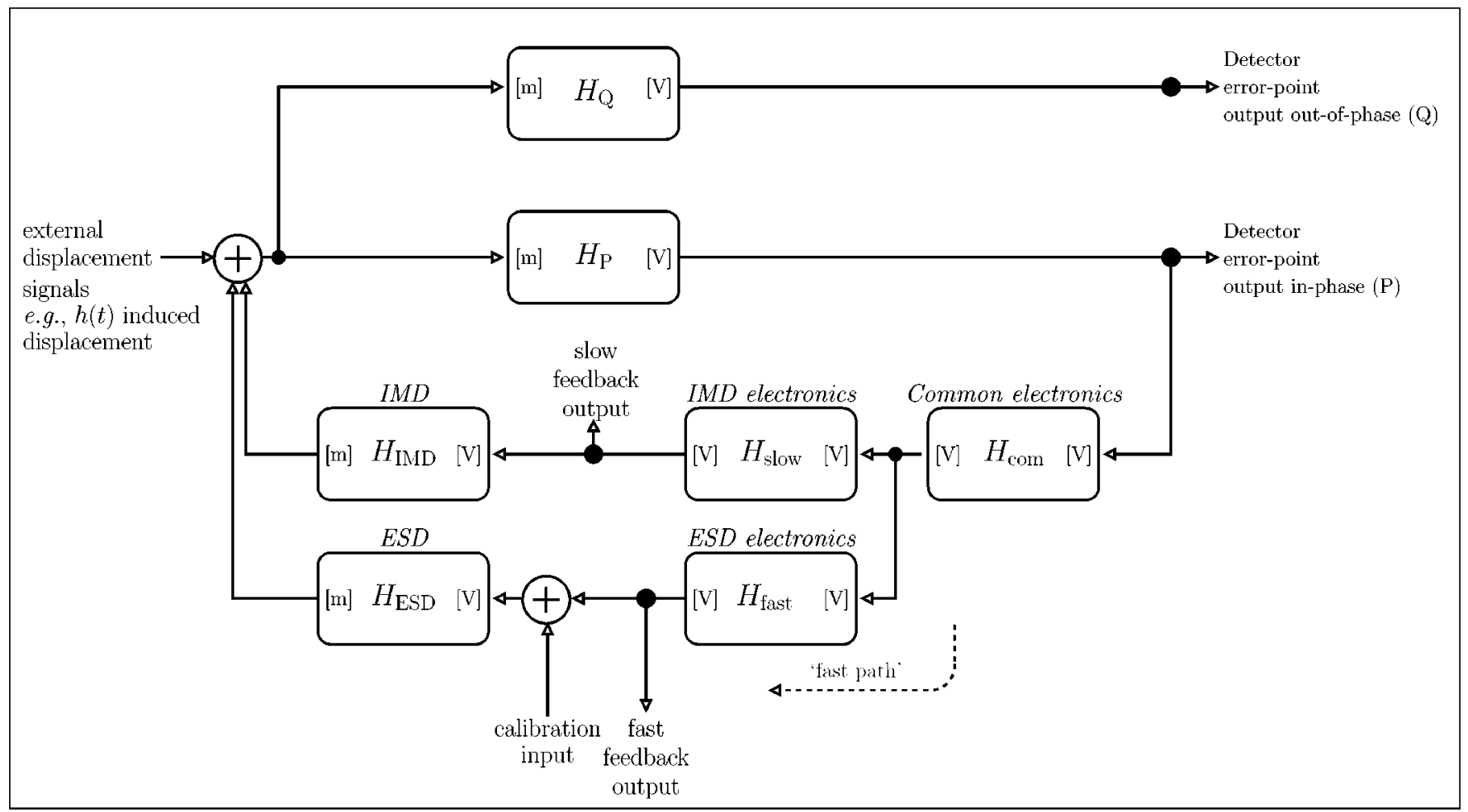

FIG. 4. A model of the length control servo of the dual-recycled GEO. Included are four relevant output signals and the input point for calibration signals. The optical response of the detector is shown as two separate transfer functions for the two demodulations, $\mathbf{P}$ and $\mathbf{Q}$.

added in the system, the SNR of a particular signal can be maintained or degraded by the presence of the noise. For example, the noise added due to the thermal noise present in the mirrors would be subject to the same signal transfer as any gravitational wave signal and so the difference between the SNRs of both quadratures would be independent of frequency. If, however, noise is added at the photon detection stage, i.e., shot noise, then the resulting difference between the SNRs of the two quadratures will be frequency dependent. For example, looking at the detector responses shown in Fig. 2, we see that low frequency gravitational wave signals will appear with a much higher amplitude in the $\mathbf{P}$ quadrature than in the $\mathbf{Q}$ quadrature. Although the recovered displacement signals will contain the same displacement information, the noise in the one recovered from the $\mathbf{Q}$ quadrature would be significantly higher at low frequencies than in the one recovered from the $\mathbf{P}$ quadrature. So for low frequencies we would want to strongly weight the $\mathbf{P}$ displacement signal and weakly weight the $\mathbf{Q}$ signal. In general, the recoverable SNR will be frequency dependent and the optimal SNR displacement signal can be achieved by summing the four (if we include the two strain signals recovered from the feedback signals) recovered displacement signals with suitable, frequency dependent, weighting functions.

The final goal of calibrating the dual-recycled GEO is to recover four calibrated displacement signals, and then (if necessary) combine them to get one displacement signal that has the optimal SNR at all frequencies in the detection band. This can be expressed mathematically as

$$
d_{\text {optimal }}=W_{\mathbf{P}} d_{\mathbf{P}}+W_{\mathbf{Q}} d_{\mathbf{Q}}+W_{\mathrm{sfb}} d_{\mathrm{sfb}}+W_{\mathrm{ffb}} d_{\mathrm{ffb}},
$$

where the frequency dependent weighting factors, $W_{*}$, must be determined from considerations of the signal-to-noise ra- tio and transfer functions of each signal. The subscripts $\mathbf{P}$ and $\mathbf{Q}$ refer to the respective quadratures, and the subscripts "sfb" and "ffb" refer to the slow (low-frequency) feedback signal and the fast (high-frequency) feedback signal, respectively. The exact form of these optimal weighting functions is yet to be determined and further investigations will be carried out when a more detailed implementation of the calibration scheme has be tried and tested.

In order to calibrate the detector, it is essential to have a good model of the relevant subsystems that directly affect the transfer of strain signals to the detector output(s). Such a model is shown in Fig. 4. Four relevant output signals are highlighted, as well as the input for the calibration signal(s); the diagram also shows the split feedback path of GEO. The model is slightly different to that used in the power-recycling calibration scheme (see Ref. 6): the part of the model that imitates the servo electronics has been split into three parts (previously two) to better mimic the real electronics.

The problem of calibrating the dual-recycled interferometer can be more easily approached by splitting up the problem into smaller subprocesses. Figure 5 shows how the problem can be split into four separate tasks that, together, lead to a calibrated strain signal. Each of the main tasks will be discussed in detail in the following sections.

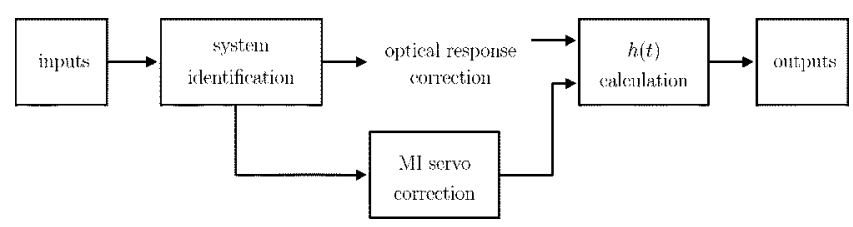

FIG. 5. A schematic of the tasks involved in the calibration of the dualrecycled GEO. 


\section{A. System identification}

The aim of this process is to determine the parameter set that best describes the detector at any particular instant in time. In the power-recycled calibration scheme this task (though not named as such) only determined the optical gain of the detector. In the case of calibrating the dual-recycled interferometer, the minimum parameter set that must be determined consists of the 4 (or 8 if the $\mathbf{Q}$ quadrature is included) optical parameters described in Sec. II C 1. In principle, this parameter set can be extended to include the other gains in the system: two electronic gain settings and the two actuator calibrations.

If we are to correct for any fluctuations in the underlying parameters of the system, then we have to estimate the parameters on similar time scales as the fluctuations occur. The current scheme, as was the case for the calibration of the power-recycled GEO, assumes we can inject calibration lines that will have a detectable SNR of around $40 \mathrm{~dB}$ in the high-power error-point using one second of data. (The amplitude with which we can inject the calibration lines is limited by the dynamic range of the feedback actuator.) Typically, this is easy to achieve and it is possible to make lownoise parameter estimations at $1 \mathrm{~Hz}$. The remainder of this text assumes measurements of calibration lines and subsequent parameter estimations occur at $1 \mathrm{~Hz}$.

The approach chosen for estimating the parameter set is to sample the underlying transfer function from differential mirror motion to demodulated detector output ( $\mathbf{P}$ and/or $\mathbf{Q}$ ) at a number of different frequencies and then to fit a model of the transfer function to these data points using a leastsquares optimisation routine. For the remainder of this discussion we will consider only the parameters for $\mathbf{P}$; the determination of the $\mathbf{Q}$ parameters can be done in the same way using the appropriate model of the optical response.

\section{Continuous transfer function estimation}

Estimation of the optical transfer function is done by measuring the magnitude and phase of the calibration lines in both the injected calibration signal and the recorded demodulated error-point signal $(\mathbf{P})$. From these measurements we get a set of complex numbers, $\mathbf{C}_{f}$ from the calibration signal, and $\mathbf{P}_{f}$ from the $\mathbf{P}$ error-point signal (where the subscript $f$ denotes the frequency of the calibration line being measured).

From Fig. 4 we can see that the transfer function, $T_{\mathrm{CP}}$, from calibration input to $\mathbf{P}$ can be written as

$$
T_{\mathrm{CP}}(s)=H_{\mathrm{ESD}}(s) H_{\mathbf{P}}(s) \operatorname{CLTF}(s),
$$

where $\operatorname{CLTF}(s)$ is the closed-loop transfer function of the servo.

The closed-loop transfer function can be written as

$$
\operatorname{CLTF}(s)=\frac{1}{1+\operatorname{OLG}(s)},
$$

where the open-loop gain, $\mathrm{OLG}(s)$, is given by

$$
\begin{aligned}
\mathrm{OLG}(s)= & H_{\mathbf{P}}(s) H_{\text {com }}(s)\left[H_{\text {slow }}(s) H_{\mathrm{IMD}}(s)\right. \\
& \left.+H_{\text {fast }}(s) H_{\mathrm{ESD}}(s)\right] .
\end{aligned}
$$

From the measurements of the calibration lines we can then form the transfer function

$$
T_{\text {measured }_{f}}=\frac{\mathbf{P}_{f}}{\mathbf{C}_{f} \mathbf{E}_{f}},
$$

where the values $\mathbf{E}_{f}$ are taken from a model of the electrostatic drive actuator, $H_{\mathrm{ESD}}$. This transfer function is the transfer function from differential arm-length change to $\mathbf{P}$.

From Eq. (5) we can see that this measured transfer function is equivalent to the transfer function $T_{\text {model }}$ $=H_{\mathbf{P}}(s) \operatorname{CLTF}(s)$ for the correct choice of parameters. Using an optimization routine, we can determine the best parameter set that matches the model transfer function, $T_{\text {model }}$ to the measured transfer function, $T_{\text {measured }}$.

If we continuously inject a sufficient number of calibration lines (more than the number of parameters we try to recover), then we can estimate the underlying parameters of the detector at least once per second. To do this, we must have a frequency-domain model of the Michelson servo loop, including a full model of the electronics. We have already discussed a possible model of the optical response of the detector in Sec. II C 1; the models for the two actuators are essentially those used in the case of calibrating the power-recycled interferometer (with appropriate calibration factors). As the detector is still in the commissioning phase (during which time the Michelson locking-servo electronics change regularly), models of the electronics need to be generated for the particular data segment being calibrated.

\section{B. Correcting for the optical response of the detector}

The optical response of the detector can be considered conceptually as two separate responses. Looking at Eq. (2), we see that it can be split into a varying gain factor that is flat in frequency, and a frequency dependent response that has fixed overall gain but varying frequency response. The parameters that describe both parts are obtained in the system identification routine. To correct the detector output in the time domain, we need to divide the output signal by the flat gain factor and then filter the result through the inverse of the frequency dependent part. When the error-point signal is "corrected" in this way, it represents (at least at frequencies where there is no loop gain) the differential displacement of the end mirrors of the interferometer. Stating this mathematically, we can write the correction for the flat timevarying gain as

$$
P_{\text {opt }}[n]=\frac{P[n]}{G_{\mathbf{P}}[n]},
$$

where $n$ is the sample being processed, $P[n]$ are the recorded samples of the high-power error-point signal, $\mathbf{P}$, sampled at $16384 \mathrm{~Hz}$, and $G_{\mathbf{P}}[n]$ are the samples of the overall optical gain factor (which we so far have at only $1 \mathrm{~Hz}$ ). From this we can recover a representation of the differential mirror displacement by

$$
d x_{\text {opt }}[n]=F_{\text {opt }} \otimes P_{\text {opt }}[n],
$$

where $\otimes$ denotes filter application, and $F_{\text {opt }}$ is a time-domain filter that has a frequency response equal to the inverse of the estimated optical response of the detector.

We can see that two problems immediately arise. First, we need a sample of $G_{\mathbf{P}}$ at each sample instant of the error 


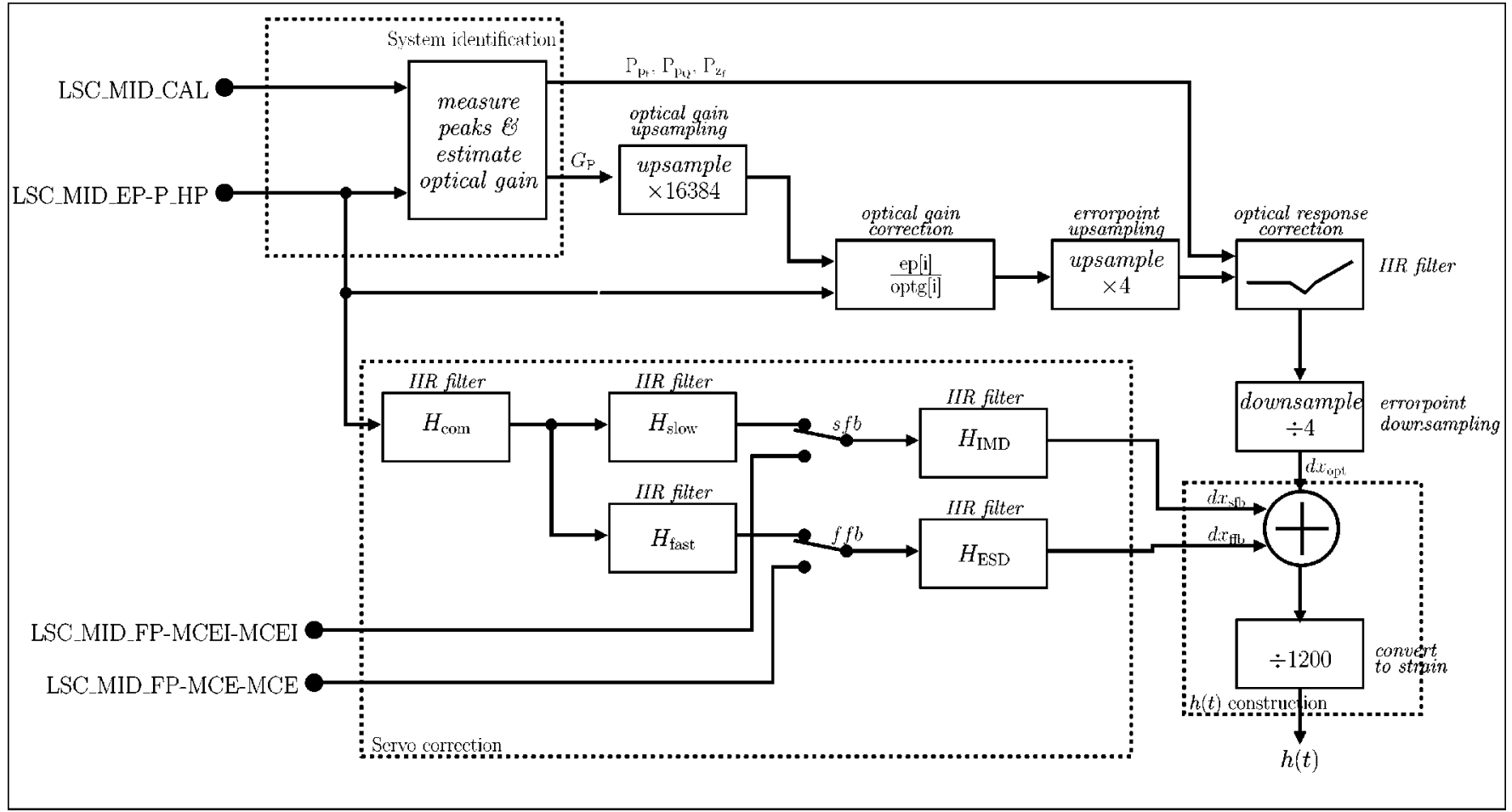

FIG. 6. A simplified schematic of the data processing pipeline for calibrating the dual-recycled GEO. The two possible loop correction procedures are shown, with "mechanical" switches representing the conceptual switch between the two methods. Input signals are labelled using their GEO DAQ labels (with the G1: prefix dropped).

point if we are to avoid discontinuities in the recovered strain signal. The error-point signal is sampled at $16384 \mathrm{~Hz}$ whereas the estimates of $G_{\mathbf{P}}$ are only obtained every second. We must therefore up-sample the estimates of $G_{\mathbf{P}}$ to 16384 Hz. This can be done by performing a band-limited interpolation. The other problem concerns the inverse of the optical response.

The inverse of the optical response will have two zeros and one pole (inverting a filter response can be considered as converting all poles to zeros, and all zeros to poles with a suitable change of gain). While such a filter would be unstable if implemented as an analog filter, it can be stable as an IIR digital filter. An IIR time-domain filter with the response of the inverse of the optical response is, however, difficult to design; the response of a filter designed using standard methods (for example, a bilinear transform) typically deviates from the desired response towards the Nyquist rate of the filter. This is not desirable since the upper frequency at which we wish to perform an accurate calibration approaches the Nyquist frequency of the sampled data. One method of dealing with this is to design the filter for a much higher sample rate of data; the response of this filter can be controlled by placing additional poles at high frequency (much higher than the band we are interested in): this avoids the introduction of numerical errors due to large amplification of high-frequency content of the signal. In order to use such a filter, the input data needs to be up-sampled. After filtering, the corrected signal can be down-sampled back to $16384 \mathrm{~Hz}$ with minimal introduction of errors.

The response of the optical-response-correction filter must adapt in time to reflect the optical parameters recovered from the system identification routine. One simple way to do this is to use the bilinear transform to analytically calculate the filter coefficients based on the estimated optical pole/zero parameters. The filter coefficients can be re-evaluated each time a new estimate of the optical response is acquired (once per second, for example). The filter can then be allowed to adapt smoothly to any changes in the optical response of the detector.

\section{Correcting for the Michelson differential length- control servo}

The principle of loop gain correction was introduced in the calibration of the power-recycled interferometer (see Sec. II A) and is no different for the case of calibrating the dualrecycled interferometer. Another possible method can also be explored that, in principle, could provide a better representation of the loop correction signals. If we look at a detailed system diagram for the calibration process (Fig. 6), we can see that there are two possibilities for recovering the signals labeled $\mathrm{sfb}$ and $\mathrm{ffb}$. One method uses the recorded errorpoint signal filtered through a model of the feedback electronics (that which was employed for the power-recycled GEO calibration scheme). While this provides a completely noise-free representation of the feedback signals, it also relies on an accurate model of the (complicated) feedback electronics. The other method is to record the feedback signals that are applied to the two actuators. This method, in principle, provides the best representation of the feedback signals, especially as it also includes any gain drifts of the electronics. However, if the electronics add any noise (or signal, 
for example, calibration lines) to the error-point signal before the point where we extract the feedback signals for recording, then the recorded feedback signals would add noise (or signals) to the recovered strain signal.

\section{Calculating the strain signal}

From the optical correction and loop correction tasks, we recover three data streams that each contain some information about the differential displacement of the end mirrors of the interferometer: $d x_{\mathrm{opt}}, d x_{\mathrm{sfb}}$, and $d x_{\mathrm{ffb}}$. These three signals, when appropriately combined, give a calibrated strain signal. This method was presented in detail in the calibration scheme for the power-recycled GEO and is stated here just for completeness. The recovered strain signal is then given by

$$
h[n]=\left(d x_{\mathrm{opt}}[n]+d x_{\mathrm{sfb}}[n]+d x_{\mathrm{ffb}}[n]\right) / 1200 .
$$

Following such a scheme can allow the development of time-domain calibration software that can calculate, in realtime, a calibrated strain signal from the output(s) of the detector.

${ }^{1}$ B. Willke et al., Class. Quantum Grav. 21, No. 5 (2004).

${ }^{2}$ K. A. Strain and B. J. Meers, Phys. Rev. Lett. 66, 1391 (1991).

${ }^{3}$ G. Heinzel et al., Phys. Rev. Lett. 81, 5493 (1998).

${ }^{4}$ G. Heinzel et al., Class. Quantum Grav. 19, No. 7 (2002).

${ }^{5} \mathrm{H}$. Grote et al., Class. Quantum Grav. 21, S473 (2004).

${ }^{6}$ M. Hewitson et al., Rev. Sci. Instrum. 74, 4184 (2003).

${ }^{7}$ M. Hewitson et al., Class. Quantum Grav. 20 (2003).

${ }^{8}$ Finesse homepage, http://www.rzg.mpg.de/ adf/ 